\title{
¿Son adecuados los desayunos que se realizan en los centros escolares?
}

\author{
Marina Alférez Corral, Patricia Trujillo Ruiz, Anabella Garzón Fernández. Universidad de \\ Almería
}

Recepción: 30 de mayo de 2016 | Revisión: 31 de mayo de 2016 | Aceptado: 1 de junio de 2016

Correspondencia: agarzon@ual.es

\begin{abstract}
Resumen: La salud de la población es un tema que implica a diferentes agentes sociales, a la comunidad educativa, a las familias, a los gobiernos, a las industrias alimentarias y al propio alumnado. Actualmente la alimentación de la población se está alejando cada vez más de las recomendaciones y costumbres alimenticias propias de nuestra cultura como es la dieta mediterránea, ocasionando problemas de salud asociados a una mala alimentación (diabetes, sobrepeso, obesidad, malnutrición, etc.) y agravadas por el aumento del sedentarismo. El objetivo de este trabajo es concienciar a las personas próximas al entorno escolar sobre la importancia de realizar una alimentación equilibrada, variada y suficiente desde la infancia, y el deber de los centros educativos en fomentar buenos hábitos en general, y en particular, en lo que respecta al desayuno que se realiza en los centros escolares. Hemos analizado los desayunos de media mañana que realizan los alumnos y alumnas de un aula de infantil y encontramos que en muchos casos no son variados, sobrepasan las necesidades calóricas y son desequilibrados en cuanto a contenido en nutrientes según las necesidades para estas edades. Sería necesario hacer llegar a los centros educativos unas pautas claras y realizar intervenciones para concienciar a las familias de la importancia de realizar un desayuno adecuado, completo y saludable.
\end{abstract}

Palabras clave: Alimentación | Educación para la Salud

Are Adequate Breakfasts that are made in Schools?

Abstract: The health of the population is an issue that involves different social actors, the educational community, families, governments, the food industry and the students themselves. Currently the supply of the population is moving increasingly from the recommendations and their own dietary habits of our culture as is the Mediterranean diet, causing health problems related to poor nutrition (diabetes, overweight, obesity, malnutrition, etc.) and aggravated by the increased sedentary lifestyle. The aim of this work is to educate the next to the school environment on the importance of a balanced, varied and sufficient food since childhood, and the duty of schools to foster good habits in general people, and in particular as Regarding the breakfast that takes place in schools. We analyzed the mid-morning breakfasts made by students in a classroom child and found that in many cases are not varied, exceed caloric needs and are imbalanced in terms of nutrient content as needed for this age group. It would be necessary to reach schools clear guidelines and interventions to raise awareness among families of the importance of a proper, full and healthy breakfast.

Keywords: Food | Health Education

\section{Introducción}

Los cambios sociales que estamos viviendo en los últimos años, están afectando directamente a nuestra alimentación y la de los más pequeños. El mundo de hoy se caracteriza por presentar mensajes contradictorios; por un lado, desde las instituciones sanitarias nos transmiten las ventajas de llevar una vida sana, y por otro, las grandes empresas alimentarias y sus mensajes publicitarios, nos incitan al consumo de comidas precocinadas y envasadas, con la consiguiente ingesta excesiva de grasas y azúcares, y la disminución de proteínas de origen vegetal, verduras y frutas, de alimentos frescos y de temporada en general. Vivimos en un ambiente denominado "obesogénico", debido a los condicionantes sociales actuales, que han dado lugar al aumento del sedentarismo y a los drásticos cambios en nuestra dieta actual. Se ha encontrado que existe relación entre unos hábitos inadecuados de niños y niñas y un peor rendimiento escolar. Un desayuno correcto es fundamental para el buen desarrollo y crecimiento del escolar, debiendo aportar los nutrientes necesarios para afrontar el día y optimizar las capacidades del alumnado. Existe certeza científica de que los hábitos alimentarios y estilo de vida que se adquieren durante la infancia perduran en el tiempo y determinan las conductas y comportamientos en la edad adulta (Pérez, 2008), por ello es crucial actuar y prevenir desde edades tempranas. La alimentación conforma el agente externo más concluyente en el crecimiento y 
desarrollo de la persona a lo largo de la infancia. Por tanto, el periodo escolar es una etapa sensible y frágil, donde se debe garantizar la correcta nutrición y afianzar las bases de un estilo de vida saludable, la promoción de la salud y el bienestar físico, mental y social.

Centrándonos en el desayuno, en primer lugar, hay que señalar la importancia de éste en la dieta de niños y adultos en general. Desde el Ministerio de Sanidad, Servicios Sociales e Igualdad, y a través de la Estrategia NAOS (Estrategia para la Nutrición, Actividad Física y Prevención de la Obesidad), en una de sus publicaciones, Guía fácil para un desayuno y una merienda saludable, Martínez (2014) afirma que:

Está demostrada la importancia del desayuno en:

- La mejora global del estado nutricional y del bienestar del niño.

- Tiene un papel determinante en el rendimiento académico.

- Está comprobado que aquellos que desayunan apropiadamente tienen menos riesgos de padecer obesidad.

- El aporte energético del desayuno es de gran importancia ya que facilitará alcanzar un adecuado rendimiento tanto físico como intelectual.

También la Asociación Española de Pediatría (AEPED), a través de su página web recoge publicaciones que defienden la importancia del desayuno, afirmando que uno de los objetivos nutricionales debe ser la promoción de un buen desayuno, ya que mejora el rendimiento intelectual, físico y la actitud en el trabajo escolar, mientras que la omisión del desayuno interfiere en los procesos cognitivos y de aprendizaje (Peña, 2002).

Según datos de un informe del Ministerio de Sanidad y Consumo, establece que sólo el $7,5 \%$ de los niños/as de nuestro país lleva a cabo un desayuno completo y equilibrado (fruta, lácteo, y cereales). Aproximadamente el 19,9\% de los niños/as y jóvenes toman únicamente un vaso de leche al levantarse, mientras que el $56 \%$ ingieren además algún alimento que les aporte hidratos de carbono y que la mitad de todos ellos no dedican más de diez minutos a desayunar. Estos datos llevan a considerar que la mayoría de los niños y niñas, tanto de educación infantil como los jóvenes españoles, no llevan a cabo un desayuno saludable y equilibrado.

En los primeros años de vida se consolidan los hábitos alimentarios y la formación del gusto por determinados alimentos, en ellos influyen tanto factores genéticos, ambientales y culturales. En el curso del proceso de socialización, el niño puede conseguir incorporar nuevos hábitos alimentarios que actúan sobre las prácticas que ya tenía previamente su familia (Hernández, 2008). La educación nutricional se inicia en el hogar, pero la escuela debe completar y educar de forma paralela, para la adquisición de unos hábitos y actitudes que favorezcan una vida saludable.

A partir de los 3 años de edad, el ritmo de crecimiento se va haciendo más lento y firme hasta que comienza el estirón debido a la pubertad. En la infancia se consolidan los hábitos alimenticios, proceso donde tienen una importancia básica la educación, tanto en el seno de la familia como en las guarderías y en las escuelas. Durante ese periodo, se adquieren conductas que marcan la vida del futuro adulto. Sin embargo, "que los niños y niñas de nuestro país no se alimentan de manera adecuada es una realidad que pocos discuten. Los análisis [...] sobre el estado nutricional de la población infantil, los programas que las administraciones sanitarias y educativas han puesto en marcha [...] son referencias importantes para intentar afrontar esta situación" (Banet y López, 2009). 


\section{El desayuno ideal en la infancia}

El desayuno, una de las comidas más importantes del día, debe aportar al menos el $25 \%$ de los nutrientes necesarios para afrontar la jornada y optimizar las capacidades de los escolares en unos momentos donde el esfuerzo físico y mental suele ser bastante intenso (Banet y López, 2009). El equilibrio de la primera comida del día favorece un reparto más armónico de las calorías que necesitamos y proporciona, también, una ración de seguridad de muchos nutrientes especialmente significativos en el período escolar, etapa de gran crecimiento y desarrollo.

El ser humano requiere una alimentación variada y equilibrada. A pesar de que uno de cada dos adultos reconoce los beneficios de tomar un buen desayuno para comenzar el día con fuerza, en Europa existen siete millones de niños que acuden al colegio cada mañana sin desayunar (Iglesias, 2008).

La ingesta de alimentos del grupo de cereales durante el desayuno es esencial, siendo la base de la alimentación diaria. De igual modo, conviene que los niños y niñas tomen alguna pieza de fruta, pudiendo ser una opción para el desayuno un buen zumo de naranja recién exprimido, aunque siempre es preferible el consumo de la pieza entera. Las frutas contienen antioxidantes, fibra, vitaminas y minerales. Es recomendable acompañar el desayuno con aceites grasos insaturados, como el aceite de oliva, que favorece la eliminación de sustancias de desecho. La leche y los lácteos son igualmente indispensables ya que aportan calcio y proteínas esenciales para el crecimiento y desarrollo del niño y de sus huesos. En cuanto a los azúcares y derivados, ofrecen un gran aporte de energía pero pocos nutrientes, por lo que aunque no se deben eliminar de la dieta, sí controlar su consumo, especialmente en la primera comida del día.

\section{Posibles causas que afectan a la alimentación del niño actual}

En los últimos años hemos presenciado un cambio progresivo, de un estilo de vida más tradicional de las generaciones pasadas, a otro con la instalación de la sociedad de consumo actual. El desarrollo y avance de la sociedad y del entorno que nos rodea termina por afectar a los agentes más susceptibles: el niño, la familia y la escuela. El ambiente familiar puede verse afectado por distintas razones, como por ejemplo, el limitado tiempo del que actualmente disponen los padres para dedicarse más y mejor a sus hijos o a las tareas de la casa, y en concreto a la alimentación.

A consecuencia de los cambios que ha sufrido la sociedad en los últimos años, es cada vez más frecuente que los niños y niñas de corta edad entren en contacto con guarderías o escuelas infantiles. Además, "el número de usuarios del comedor ha aumentado cuantiosamente como consecuencia, especialmente, de la incorporación de las mujeres al mundo laboral y la tendencia a constituir el lugar de residencia a las afueras de la ciudad" (Aranceta, 2008). La familia debe tratar de organizar su tiempo para que el escolar pueda disfrutar de un desayuno adecuado. Las prisas por llegar a tiempo al colegio y la somnolencia que acarrean los más jóvenes en los primeros momentos de la mañana impiden, en algunos casos, tomar un desayuno completo y nutritivo.

\section{Sugerencias para alcanzar unos hábitos saludables en la infancia}

Aunque cada vez está más vigente la idea del cuidado personal, la alimentación saludable y una vida activa para mejorar nuestra salud, aún queda mucho trabajo por realizar, principalmente, aumentar el grado de concienciación en todos los hogares. 
Abordar los problemas alimenticios hoy en día es bastante complicado. Esto es debido a la cantidad de estímulos mediáticos a los que estamos sometidos, enfocados a despertar en la sociedad nuevos deseos y necesidades. Por ello, es fundamental que la familia procure mantener una vida sana y activa, unos buenos hábitos que promuevan su bienestar.

La familia debe integrar en su vida diaria actividades físicas, además de ser una buena manera para inculcar valores y hábitos saludables en el niño, también lo es para afianzar y reforzar la relación padres-hijos, aumentar el gasto de energía del cuerpo, ayudar a controlar el apetito, mejorar la imagen personal de uno mismo, la autoestima y el estado de ánimo.

El colegio también marca unas rutinas en torno a las cuales se consolidan la mayoría de los hábitos que adquirimos en la vida. El centro escolar es un lugar significativo para la adquisición de conocimientos prácticos y teóricos sobre la alimentación y la salud, ya que los niños pasan la mayor parte del tiempo en él. Es por tanto, un pilar fundamental en la prevención de enfermedades y modificación de hábitos alimenticios. El comedor escolar, desempeña además una función social y formativa bastante importante con un alto grado de responsabilidad.

\section{Objetivos}

- Concienciar a las personas próximas al entorno escolar sobre la importancia de una alimentación saludable desde la infancia, centrándonos en el desayuno en la edad escolar.

- Analizar los desayunos que realizan a media mañana los niños de infantil.

\section{Método}

\section{Participantes}

La muestra que participó ha participado en la investigación se corresponde con un grupo de 27 alumnos y alumnas de la etapa de infantil de 5 años, de un centro escolar de Almería.

\section{Instrumentos}

Para llevar a cabo el estudio, se ha realizado una toma de datos, mediante observación directa, de los desayunos que traen los niños al colegio y durante quince días (3 semanas, de lunes a viernes). Se ha calculado el aporte calórico de dicho desayuno de media mañana, usando el etiquetado nutricional del producto o realizando un cálculo aproximado según tablas de composición de alimentos y teniendo en cuenta el tamaño de la ración/porción. Se ha comparado dicho aporte calórico con el recomendado según la edad de los niños/as. El grado de bondad de los desayunos se ha analizado desde un punto de vista cuantitativo como cualitativo. Desde el punto de vista cuantitativo, se ha determinado si los desayunos que traen los niños cumplen con los requerimientos de energía necesarios que debería aportar esa comida en ese momento del día, teniendo en cuenta la edad y nivel de actividad de los niños.

Para determinar el requerimiento energético necesario para un niño de 5 años, se ha realizado una revisión bibliográfica sobre dichos valores. Según Peña, Ros, González, Rial (2010) las necesidades energéticas para niños y niñas de entre 3-6 años y según nivel de actividad serían los que se presentan en la siguiente Tabla 1. 


\begin{tabular}{|c|c|c|c|c|c|c|c|c|}
\hline & \multicolumn{8}{|c|}{ Tabla 1. Requerimientos energéticos estimados (Kcal/día) ${ }^{*}$} \\
\hline & \multicolumn{2}{|c|}{$\mathrm{NAF}^{* *}$ sedentario } & \multicolumn{2}{|c|}{$\mathrm{NAF}^{* *}$ activo-bajo } & \multicolumn{2}{|c|}{$\mathrm{NAF}^{* *}$ activo } & \multicolumn{2}{|c|}{$\mathrm{NAF}^{* *}$ muy activo } \\
\hline & Niño & Niña & Niño & Niña & Niño & Niña & Niño & Niña \\
\hline 3 & 1.160 & 1.100 & 1.300 & 1.250 & 1.500 & 1.400 & 1.700 & 1.650 \\
\hline 4 & 1.200 & 1.130 & 1.400 & 1.300 & 1.575 & 1.475 & 1.800 & 1.750 \\
\hline 5 & 1.275 & 1.200 & 1.470 & 1.370 & 1.650 & 1.550 & 1.900 & 1.850 \\
\hline 6 & 1.300 & 1.250 & 1.500 & 1.450 & 1.750 & 1.650 & 2.000 & 1.950 \\
\hline \multicolumn{9}{|c|}{ 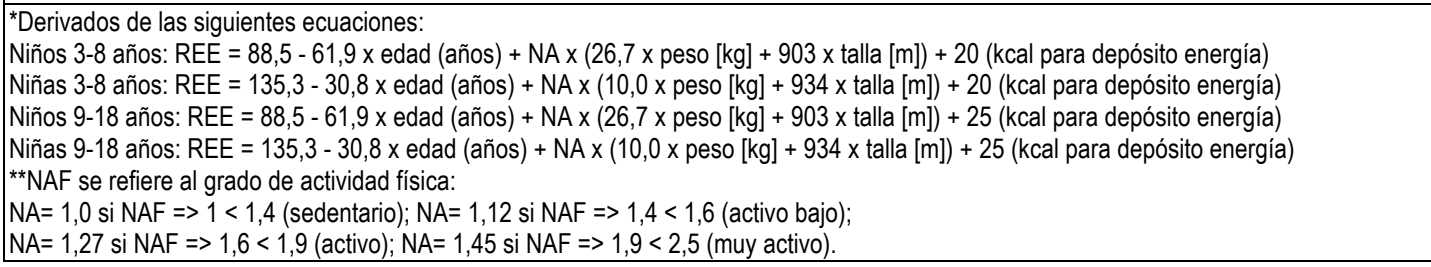 } \\
\hline
\end{tabular}

Según Peña (2002), para los niños de 4 a 6 años de edad, sin tener en cuenta el nivel de actividad física estableció, en general, un requerimiento energético de unas 1.800 $\mathrm{kcal} / \mathrm{día}$.

Según datos de la Dietary guidelines for Americans (2005), los requerimientos de energía estimados (en kilocalorías) para cada categoría (género y edad) y teniendo en cuenta tres niveles de actividad física, serían los siguientes:

\begin{tabular}{|c|c|c|c|c|}
\hline & & \multicolumn{3}{|c|}{ Nivel de actividad } \\
\hline Género & Edad (años) & Sedentario & Moderadamente activo & Activo \\
\hline Niño/a & $2-3$ & 1.000 & $1.000-1.400$ & $1.000-1.400$ \\
\hline Niña & $4-8$ & 1.200 & $1.400-1.600$ & $1.400-1.800$ \\
\hline Niño & $4-8$ & 1.400 & $1.400-1.600$ & $1.600-2.000$ \\
\hline
\end{tabular}

Según datos de la Food and Nutrition Board. Institute of Medicine of the National Academies, (2005), para niños de entre 3-8 años las necesidades de energía serían unas $1742 \mathrm{Kcal} /$ día y para las niñas unas $1642 \mathrm{Kcal} / \mathrm{día}$. Sin embargo para Ortega, Requejo, Navia y López-Sobaler (2014), las ingestas diarias recomendadas de energía en la etapa preescolar (4-5 años) serían unas $1600 \mathrm{kcal}$. Por tanto, encontramos que dependiendo de las fuentes los valores pueden variar, estando las necesidades energéticas 1500-1800 Kcal/día.

En cuanto al aporte energético que debe constituir el desayuno, entendiendo que el mismo incluye tanto el desayuno en casa como el desayuno a media mañana que se realiza en el colegio, también puede haber pequeñas diferencias.

Peña (2002), establece para los niños de 4 a 6 años de edad el siguiente reparto calórico entre las diferentes comidas del día: $25 \%$ en el desayuno, $30 \%$ en la comida, $15 \%$ en la merienda y $30 \%$ en la cena.

La Guía fácil para un desayuno y una merienda saludable, recogida en Publicaciones NAOS, Martínez (2014), establece la siguiente distribución: Desayuno 20\%, Almuerzo de media mañana $10-15 \%$, Comida $25-35 \%$, Merienda $10-15 \%$, Cena $25 \%$.

Otros autores afirman que conviene establecer un orden con 4-5 comidas/día, distribuyendo las calorías a ingerir entre el desayuno (20-25\% del total), comida (30$35 \%)$, merienda (15-20\%) y cena (25\%) (Ortega, Jiménez, Perea y Navia, 2014). 
Según revisión bibliográfica, los valores de la distribución de aportes calóricos diarios, pueden variar entre:

- Un $20-25 \%$ de las kcal en el desayuno (ó 15\%+10\% media mañana).

- Un $30-35 \%$ en la comida del mediodía.

- Un $10-15-20 \%$ en la merienda.

- Un $20-25-30 \%$ en la cena.

Por lo tanto, y a partir de los datos expuestos, podríamos considerar que el aporte calórico necesario para los niños de la clase de 5 años debería ser, aproximadamente, de unas 1.500-1.800 kcal/día. El desayuno de media mañana que se realiza en el colegio debería aportar aproximadamente un $10 \%$ de dicho requerimiento energético, esto es, entre $150-180 \mathrm{kcal} / \mathrm{día}$.

\section{Procedimiento}

La recogida de datos se llevó a cabo durante 15 días entre el mes de noviembre y diciembre, equivalente a tres semanas de clase, de forma manual, mediante la utilización de una tabla de registro, apuntando junto al nombre de cada niño y lo que había traído para desayunar ese día. Se ayudó también mediante la realización de fotos del etiquetado. Esto facilita la toma de datos ya que muchos alimentos, sobre todo las bebidas se repiten, así que se pueden construir tablas de recogida de información en las que esos alimentos aparezcan ya escritos y solamente haya que marcar una cruz para confirmar que se han traído, o el nombre del producto.

\section{Resultados}

El aporte calórico del desayuno de media mañana supera en muchos casos y en la mayoría de los días, el límite calórico para lo que debiera ser esta comida del día (150$180 \mathrm{kcal}$ ). Se ha registrado el aporte calórico de los alimentos que forman parte habitualmente del desayuno de media mañana, de acuerdo con los datos recogidos, a partir de la información nutricional contenida en el etiquetado de los propios alimentos o en su defecto realizando un cálculo aproximado según tablas de composición de alimentos. (Ver anexo Tabla 1. Composición calórica de los alimentos consumidos por los alumnos).

Para calcular el contenido calórico de los desayunos de media mañana, combinamos una bebida con un alimento sólido, que es lo que suelen traer los niños al colegio.

Tabla 1. Ejemplos de desayunos de media mañana ( $k$ cal totales del desayuno).

\begin{tabular}{|c|c|c|c|c|c|c|c|c|c|c|}
\hline & \multicolumn{2}{|c|}{ Bocadillo/sandwich } & \multicolumn{4}{|c|}{ Galletas } & \multicolumn{2}{|r|}{ Cereales } & \multicolumn{2}{|c|}{ Fruta } \\
\hline & Jamón York/Pavo & Salchichón/Chorizo & Chips Ahoy & Chiquilín & Dino/Oceanix & Gracy & Príncipe & 30 gramos & Manzana & Plátano \\
\hline Batido zumo+leche & 238 & 325 & 266 & 208 & 250 & 225 & 430 & 181 & 130 & 162 \\
\hline Batido & 284 & 371 & 312 & 254 & 296 & 271 & 476 & 227 & 176 & 208 \\
\hline Zumo & 256 & 343 & 284 & 226 & 268 & 243 & 448 & 199 & 148 & 180 \\
\hline Actimel & 260 & 347 & 288 & 230 & 272 & 247 & 452 & 203 & 152 & 184 \\
\hline Petit beber & 267 & 354 & 295 & 237 & 279 & 254 & 459 & 210 & 159 & 191 \\
\hline
\end{tabular}

\section{Discusión}

Podemos ver claramente cómo en líneas generales, el desayuno de media mañana supera el límite orientativo de $180 \mathrm{kcal} / \mathrm{d} i ́ a$ que habíamos calculado en el apartado 
anterior. Esto no significa que el total de kcal/diaria supere el límite recomendado, porque puede ser que se esté desplazando parte del desayuno que debería realizarse en casa, hacia el desayuno de media mañana en el colegio. Esto es un error, ya que puede afectar al rendimiento de los niños en las tareas de clase, más aún porque antes del recreo se realizan generalmente actividades relacionadas con el lenguaje y las matemáticas ya que durante estas horas los niños suelen estar más concentrados.

Vemos, además, como las combinaciones más ajustadas $y$, por otra parte, las más saludables son las que combinan una pieza de fruta o cereales con una bebida.

En la mayoría de los casos, para equilibrar el desayuno de media mañana bastaría con sustituir el zumo o batido por agua, que los bocadillos fueran de alimentos más magros (pavo, queso fresco, jamón, atún...) y no abusar de galletas con chocolate o bollería industrial o incluyendo más frutas a lo largo de la semana.

Pero existen algunos casos en los que el superávit calórico se evidencia todavía más, y es en los desayunos con bocadillo de embutido graso (salchichón, chorizo...) y en el caso de las galletas de chocolate. Ambos alimentos deberían consumirse con moderación.

En la clase objeto de estudio hay 4 niños ( 3 niños y una niña) con evidentes problemas de sobrepeso, lo que representa un porcentaje sobre el total (27 alumnos) del $15 \%$.

En cuanto al porcentaje de seguimiento de las recomendaciones del desayuno saludable, se ha calculado al final de cada columna, excepto para los viernes en los que el desayuno es libre. Podemos ver cómo dicho porcentaje varía desde un $42 \%$ hasta un $93 \%$, manteniéndose en general por encima del $60 \%$, salvo algunos días, lo que pone de manifiesto que las recomendaciones sobre los Desayunos Saludables, llevadas a cabo por el colegio son tenidas en cuenta por un gran porcentaje de padres a la hora de preparar el desayuno que sus hijos llevan a clase.

El aporte calórico del desayuno que los niños traen a clase es excesivo, si se calcula como el $10 \%$ del aporte calórico diario recomendado para esta comida del día. No tenemos datos del desayuno realizado en casa de todos los días y niños, pero en general suelen desayunar un vaso de leche con cacao y algún alimento del grupo de los cereales (galletas, pan, magdalenas, bollería, cereales). Teniendo en cuenta que el $25 \%$ de la energía debería aportar en total el desayuno, se correspondería con unas 400-450 kcal. En muchos casos puede que se esté desplazando parte del porcentaje recomendado del desayuno que debe realizarse en casa hacia el desayuno que se lleva a cabo en clase, tomando en casa sólo un vaso de leche con cacao, otros lácteos (yogur líquido, batidos,...) o zumos.

\section{Conclusiones}

Hoy en día, la amplia oferta de alimentos elaborados y precocinados en los grandes supermercados, gana la batalla frente a las redes de distribución de los productos frescos (frutas, verduras, pescados, carnes). El aumento de este mercado, su gran variedad, el bajo precio, su comodidad, su palatabilidad, aunque de menor calidad nutricional, supone una tentación a la hora de tomar decisiones sobre los que se compra, lo que se come. Las grandes empresas alimentarias y el acoso de la publicidad, hacen que hasta ahora estemos perdiendo la batalla de la concienciación de la población de la importancia de cuidar su salud a través de unos hábitos saludables. 
El papel de los anuncios televisivos y la publicidad ejerce también gran influencia en la alimentación saludable en edad escolar, siendo una de las causas directas que afectan a este ámbito. La publicidad, en ocasiones, nos ofrece productos poco o nada saludables para los escolares. Zumos comerciales con exceso de azúcares, bollería industrial con un alto contenido de grasas, azúcares, aditivos, ... alimentos listos para llevar y fáciles de masticar.

Las recomendaciones realizadas por el colegio sobre los "Desayunos Saludables" son seguidas por un amplio número de padres, pero en algunos casos las recomendaciones no están revisadas por ningún personal sanitario 0 con conocimientos en dietética o nutrición.

En los últimos años hemos presenciado un cambio radical en nuestro estilo de vida y alimentación, que ha terminado por afectar a los agentes más susceptibles, los niños/as. Como resultado de este cambio, encontramos consecuencias de esos estilos de vida poco saludables a nivel escolar, afectando principalmente al rendimiento académico del niño, a la calidad del sueño, a su estado de humor, a su nivel de atención, a su autoestima, etc.

La alimentación infantil es un tema que nos concierne a todos. Porque para mejorar la salud de nuestra sociedad, es preciso que todos los sectores implicados (la comunidad educativa, las familias, los gobiernos, las autoridades sanitarias, las industrias alimentarias, etc.), contribuyan a fomentar una dieta más saludable para la prevención de deficiencias y desequilibrios en la infancia.

La alimentación y el nivel de actividad de los niños en la etapa preescolar y escolar son fundamentales para establecer las bases de su salud futura. Profundizar en el conocimiento de la problemática actual (obesidad, sedentarismo, malnutrición, diabetes) y tomar medidas para la prevención supondrá un beneficio para la sanidad y para la salud de todos.

\section{Referencias}

Aranceta, J. (2008). Realidad actual de los comedores escolares en España. En Martínez, Á.J.R. y Polanco, A.I. (Eds.), El libro blanco de la alimentación escolar. (pp. 1-3). Madrid: McGraw-Hill.

Banet, E. y López, C. (2009). Educación para la sostenibilidad y la salud, 71, 63-83.

Hernández, M. (2008). Alimentación en la primera infancia. En Hernández, M. y Sastre, A. (Eds.), Tratado de Nutrición (pp. 809-831). Madrid: Díaz de Santos.

Iglesias, C. (2008). La alimentación del escolar en su domicilio. El papel del desayuno, la merienda y la cena. En Martínez, Á.J.R. y Polanco, A.I. (Eds.), El libro blanco de la alimentación escolar. (pp. 37-51). Madrid: McGrawHill.

Institute of Medicine of the National Academies (2005). Dietary reference intakes for energy, carbohydrate, fibre, fat fatty acids, cholesterol, protein and amino acids. Food and Nutrition Board. Washington: The National Academies Press.

Martínez Álvarez, JR. (2014). Guía fácil para un desayuno y una merienda saludable [en línea]. Fundación Alimentación Saludable. http://www.naos.aesan.msssi.gob.es/naos/ficheros/investigacion/desayuno merienda saludable.pdf [Consulta 21-01-2015].

Ortega R.M., Requejo AM, Navia, B. y López-Sobaler, A.M. (2014). Ingestas diarias recomendadas de energía y nutrientes para la población española. Madrid: Universidad Complutense de Madrid.

Ortega, R.M., Jiménez, Al., Perea, J.M. y Navia, B. (2014). Desequilibrios nutricionales en la dieta media española; barreras en la mejora. Nutr Hosp; 30 (2):29-35. 
Peña Quintana, L. (2002). Alimentación del preescolar y escolar [en línea]. Protocolos diagnósticos y terapéuticos en pediatría, 5, 321-328. https://www.aeped.es/sites/default/files/documentos/3-alimentacion escolar.pdf [Consulta 21-01-2015].

Peña Quintana, L., Ros Mar, L., González Santana, D., y Rial González, R. (2010). Alimetación del preescolar y escolar [en línea]. Protocolos diagnóstico-terapéuticos de grastroenterología, hepatología y nutrición pediátrica, 297305. https://www.aeped.es/sites/default/files/documentos/alimentacion escolar.pdf [Consulta 21-01-2015].

Pérez, C. (2008). La nutrición y el comedor escolar: importancia en el rendimiento de la población escolar y su influencia sobra la salud actual y futura del colectivo. En Martínez, Á.J.R. y Polanco, A.I. (Eds.), El libro blanco de la alimentación escolar. (pp. 3-9). Madrid: McGraw-Hill.

\section{Anexos}

Tabla 1. Composición calórica de los alimentos consumidos por los alumnos (kcal por ración consumida):

- Actimel: $88 \mathrm{kcal}$ en los $100 \mathrm{~g}$

- Batido chocolate, fresa o vainilla: $112 \mathrm{kcal}$ en los $200 \mathrm{ml}$.

- Batido de zumo de frutas más leche: $66 \mathrm{kcal}$ en los $200 \mathrm{ml}$

- Bollito de leche: $151 \mathrm{kcal}$ por ración.

- Cereales (trigo inflado con azúcar, jarabe de glucosa y miel): 115 kcal por 30 g. (ración).

- Galletas chips ahoy: $200 \mathrm{kcal}$ la bolsita de $40 \mathrm{~g}$.

- Galletas tipo chiquilín: 142 kcal por 30 gramos de galletas (unas 4 galletas).

- Galletas dino: $46 \mathrm{kcal}$ por galleta (10 gramos). Cuatro galletas serían $184 \mathrm{kcal}$.

- $\quad$ Galletas gracy: $159 \mathrm{kcal}$ por bolsita (cada bolsita trae 4 galletas, $32 \mathrm{~g}$.).

- Galletas oceanix: $46 \mathrm{kcal}$ por galleta, por lo que 4 galletas $(39,2$ g. y 184 kcal.)

- Galletas oreo: $143 \mathrm{kcal}$ por $30 \mathrm{~g}$. de galletas.

- Galletas príncipe: $91 \mathrm{kcal}$ por galleta $(20 \mathrm{~g}$.). Cuatro galletas serían $364 \mathrm{kcal}$.

- Galletas tostarica: $27 \mathrm{kcal}$ por galleta, por lo que 4 galletas, $(23,6 \mathrm{~g}$. y $108 \mathrm{kcal}$.)

- Jamón york: $74 \mathrm{kcal}$ por $50 \mathrm{~g}$. (30 g. unas $44 \mathrm{kcal})$.

- L. Casei: $79 \mathrm{kcal}$ cada botellín de $100 \mathrm{~g}$

- Manzana: $64 \mathrm{kcal}$ una pieza de $100 \mathrm{~g}$.

- Osito lulú: $119 \mathrm{kcal}$ por ración.

- Pan blanco: $128 \mathrm{kcal}$ por 50 gramos.

- Pan de sándwich: $62 \mathrm{kcal}$ por rebanada. Dos rebanadas $124 \mathrm{kcal}$.

- Petit beber: $95 \mathrm{kcal}$ cada uno (90 g.).

- Plátano: $96 \mathrm{kcal}$ los 100 gramos.

- Queso de bola: $114,5 \mathrm{kcal}$ por $30 \mathrm{~g}$. para un bocadillo.

- Quesito El caserío: $37 \mathrm{kcal}$ por pieza (15,6 g.).

- Quesito mini babybel: $62 \mathrm{kcal}$ cada quesito $(20 \mathrm{~g}$.).

- Queso de untar La vaca que ríe con palitos: $103 \mathrm{kcal}$ por tarrina $(35 \mathrm{~g}$.$) .$

- Salchichón: $131 \mathrm{kcal}$ por ración de $30 \mathrm{~g}$.

- Yogur: $103 \mathrm{kcal}$ cada yogur de $125 \mathrm{~g}$

- Yogur griego: $156 \mathrm{kcal}$ por yogur de $125 \mathrm{~g}$

- Zumo de naranja: $84 \mathrm{kcal}$ los $200 \mathrm{ml}$ 